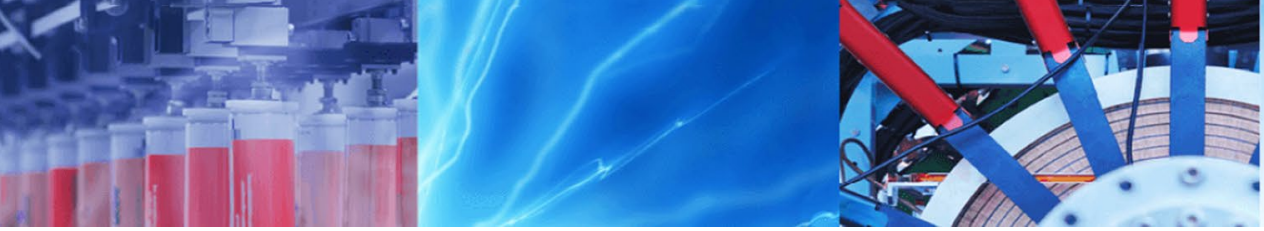

Research Article

\title{
Simulation study on ride comfort enhancement for a heavy duty vehicle with fractional order sliding mode controller
}

\author{
T. Yuvapriya ${ }^{1}$ (D) P. Lakshmi ${ }^{1}$
}

Received: 30 December 2019 / Accepted: 27 July 2020 / Published online: 5 August 2020

(c) Springer Nature Switzerland AG 2020

\begin{abstract}
The main goal of vehicle active suspension system is to upgrade the ride quality and stability of heavy duty vehicle (HDV) regardless of road surface. The mathematical modelling of HDV with two degrees of freedom is developed and simulated in MATLAB/Simulink environment. The simple and effective control techniques such as proportional integral and derivative (PID) controller, sliding mode controller (SMC) and fractional order sliding mode controller (FOSMC) are designed to detach the vibration from the passenger under step, sinusoidal and random road profile. Literature reveals that the FOSMC can eliminate steady state error and chattering effect unlike SMC and PID. The dynamic analysis of designed controllers is examined in terms of time domain, frequency domain and ride comfort analysis as per ISO 2631. The stability of SMC and FOSMC is confirmed by Lyapunouv stability for three different road profiles. The FOSMC shows satisfactory simulation results than SMC, PID and passive suspension system in reducing suspension deflection and body acceleration of HDV to upgrade the ride quality. Further, the efficacy of proposed FOSMC is proved by comparing the simulation results against existing results of PID controller.
\end{abstract}

Keywords FOSMC $\cdot \mathrm{HDV} \cdot$ Ride comfort analysis - Stability $\cdot$ VASS

\section{Introduction}

In the transportation field, tractor trailers, trucks, heavy trucks, trailer trucks and military trucks are predominantly used as heavy duty vehicles (HDVs). The continuous rise in vehicle loads and road traffic, there is a damage of vehicle parts. The passenger safety and ride comfort are greatly affected by vibration of the vehicle. In the earlier days, Passive Suspension System (PSS) is used and it does not provide travel comfort and safety for the passenger due to fixed values of spring and damper. The complications raised from PSS are moderately dealt with Semi ASS using variable damper. Hence the automotive industry introduces Active Suspension System (ASS) to enhance the ride comfort and suppresses the vehicle vibration by the actuator force. The front suspension in the midi bus reduces the intensity of rollover and improves the stability of the vehicle [1]. In mining vehicles, ride comfort can be achieved with hydraulic interconnected suspension system which provides soft bounce and decreases the pitch motion [2]. The electro hydraulic damper is utilised to improve the handling performance of heavy trucks [3]. Further, the off road vehicle dynamics are studied to forecast the vibration of the vehicle [4]. For better safety and passenger comfort, real time damped shock absorber is introduced in off-road vehicle [5].

The seat vibration in HDV can be suppressed with static output feedback $\mathrm{H}$-infinity controller and it replaces conventional linear actuator by active seat suspension system [6]. The semi active and active damper is introduced to analyse the dynamics of tractor for road-3 random road profile [7]. The higher order sliding mode controller (SMC)

T. Yuvapriya, tyuvapriya3@gmail.com; P. Lakshmi, p_lakshmi@annauniv.edu|'Department of Electrical and Electronics Engineering, College of Engineering, Guindy, Anna University, Chennai, India. 
is developed to avoid the risk of rollover and twisting algorithm is implemented to guarantee the stability of the vehicle [8]. The active rollover control technique is highlighted in HDV to reduce accidents [9]. The payload changes can significantly affect the dynamics of HDV. Hence the Linear Quadratic Regulator (LQR) is designed to upgrade the dynamics of front and rear loaded vehicle [10].

The various control strategies such as bat optimized proportional integral and derivative (PID) controller [11], fuzzy PID controller [12] and single neuron PID [13] are presented to reduce the effects of vibration in the HDV. The SMC [14], Fractional Order SMC (FOSMC) [15] and fractional order terminal SMC $[16,17]$ are designed to arrest the vibration for quarter car and full car model which enhances the travel comfort of the passenger under irregular road profile. The speed control of HDV is foremost criteria to avoid the risk of accidents. To achieve superior speed regulation performances in HDV, an improved internal model controller [18] and neural based fuzzy inference control systems [19] are presented. Based on the driving environment, drivability is evaluated by frequency weighted function and Root Mean Square (RMS) values [20]. On the other hand, available control techniques [21, 22] can improve steady state performance, while their transient behaviour such as overshoot and settling time cannot be dictated. To achieve safety and ride comfort, it is more desirable to guarantee both steady state and transient response.

Influenced by the literature survey, this paper is focussed on the design of FOSMC for HDV. The prominent factor of the proposed controller is to assure both steady state and transient response. Another key factor is to eliminate chattering effect and guarantees the stability for HDV. The prerequisites of suspension performances such as Body Acceleration (BA) and Suspension Deflection (SD), Frequency Weighted RMS (FWRMS) and Vibration Dose Value (VDV) are also discussed.

The organisation of the proposed work is as follows; in Sect. 2, the modelling equations of HDV are derived. The control strategies are presented in Sect. 3. In Sect. 4, the performances of the designed controllers are evaluated in terms of numerical and simulation results. Finally, conclusions are outlined in Sect. 5.

\section{Mathematical modelling of heavy duty vehicle}

A HDV is a complex vibration system. It is quite troublesome to develop a perfect model to reflect the complete vibration characteristics of the HDV. Further analysis, the mathematical model of HDV is accomplished to simplify

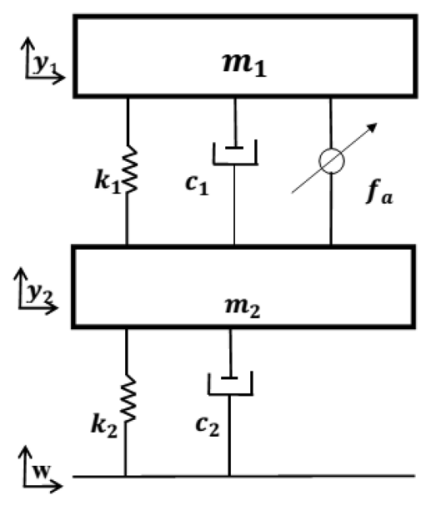

Fig. 1 Heavy duty vehicle model

Table 1 HDV parameters

\begin{tabular}{lll}
\hline Symbol & Description & Values \\
\hline $\mathrm{m}_{1}$ & Body mass & $2500 \mathrm{~kg}$ \\
$\mathrm{~m}_{2}$ & Suspension mass & $320 \mathrm{~kg}$ \\
$\mathrm{k}_{1}$ & Suspension system spring constant & $80,000 \mathrm{~N} / \mathrm{m}$ \\
$\mathrm{k}_{2}$ & Spring constant of wheel and tyre & $500,000 \mathrm{~N} / \mathrm{m}$ \\
$\mathrm{c}_{1}$ & Suspension system damping constant & $350 \mathrm{~N} \mathrm{~s} / \mathrm{m}$ \\
$\mathrm{c}_{2}$ & Damping constant of wheel and tyre & $15,020 \mathrm{~N} \mathrm{~s} / \mathrm{m}$ \\
$\mathrm{w}$ & Wheel & \\
$\mathrm{f}_{\mathrm{a}}$ & Control force & \\
\hline
\end{tabular}

the vibration system based on the research objective. The mathematical model of any system describes the relationships between the input and output along with the elements present in the system. A basic quarter HDV model with 2 DOF [22] is shown in Fig. 1. The tyre stiffness and suspension spring stiffness are assumed to be linear in their operating range and that the tyre does not leave the ground. The mathematical modelling of the HDV is done by using MATLAB.

The differential equations of quarter HDV with 2 DOF [22] are as follows;

$$
\begin{aligned}
& m_{1} \ddot{y}_{1}+k_{1}\left(y_{1}-y_{2}\right)+c_{1}\left(\dot{y}_{1}-\dot{y}_{2}\right)=f_{a} \\
& m_{2} \ddot{y}_{2}-k_{1}\left(y_{1}-y_{2}\right)-c_{1}\left(\dot{y}_{1}-\dot{y}_{2}\right)-c_{2}\left(\dot{w}-\dot{y}_{2}\right)-k_{2}\left(w-y_{2}\right)=-f_{a}
\end{aligned}
$$

The parameters of HDV [21,22] are given in Table 1.

\section{Controller design}

To suppress vibration in HDV, PID, SMC and FOSMC are designed for SASS. 


\subsection{PID controller design}

The basic form of PID control is given as

$u(t)=K_{p} e(t)+K_{l} \int e(t) d t+K_{D} d e(t) / d t$

where $\mathrm{K}_{\mathrm{p}}$, proportional gain; $\mathrm{K}_{\mathrm{i}}$, integral gain; $\mathrm{K}_{\mathrm{d}}$, derivative gain; $e(t)$, error and $u(t)$, controller output.

The aim of PID controller combines the useful characteristics of Proportional and Integral (PI) controller and Proportional Derivative (PD) controller. The output response of PID controller has minimum overshoot and return back to set point more shortly than the other forms of PI and PD controller. For higher order process, PID is necessary to prevent large overshoot and long settling time [23] and the PID controller tuning values are considered from [22]. The advantage of using this controller is to reduce the vibration to zero and overshoot.

\subsection{Sliding mode controller design}

To achieve desired ride quality and stability, SMC is designed for HDV. The designed SMC is tested for step, random and sinusoidal road disturbances. The Lyapunouv function used for $S M C$ is $V=1 / 2 s^{2}$. A necessary condition for existence and reachability of a sliding surface is $S(X, t)$ in the system state space is to choose the control variable $u(t)$ such that the following condition is satisfied

$\dot{V}=s \dot{s}<0$

The suspension deflection $\left(x_{1}\right)$ and car body velocity $\left(x_{2}\right)$ is chosen as state variables for HDV to design SMC $[24,25]$. The vibration of the HDV is decreased by applying control force generated from actuator. This control force produces opposite force to suppress vibration developed due to road disturbances.

The following sliding surface is chosen as to design SMC,

$s=x_{2}+\lambda x_{1}$

where $\lambda$ is the gain of sliding surface. By differentiating the Eq. (5),

$\dot{s}=\dot{x}_{2}+\lambda \dot{x}_{1}$

The system moves on sliding state at $\dot{s}=0$,

$0=\dot{x}_{2}+\lambda \dot{x}_{1}$

The following state variables are used to obtain the control force,

$x_{1}=y_{1}-y_{2}$ $x_{2}=\dot{y}_{1}$

The state variables are substituted in the above Eq. (6)

$f_{a}=k_{1}\left(y_{1}-y_{2}\right)+c_{1}\left(\dot{y}_{1}-\dot{y}_{2}\right)-\lambda m_{1}\left(\dot{y}_{1}-\dot{y}_{2}\right)-k \operatorname{sign}(s)$

where $\mathrm{k}$ is a positive constant and the switching function is ksign(s)

The main drawback incorporated with SMC is the discontinuity of the controller around the sliding surface $s(X, t)$. Hence the chattering effect is developed which may leads to unpredictable degradation in the system performance.

\subsection{Fractional order sliding mode controller design}

To overcome the chattering phenomenon, fractional order is included with SMC. To decrease the maximum peak overshoot and steady state error, the FOSMC is developed to improve the transient behaviour of the HDV. The state variables for designing FOSMC are chosen from SMC. The FOSMC is designed by including fractional derivative term in the SMC equation [24]. The sliding surface for FOSMC is chosen as

$s=\mathrm{D}^{\alpha-1} x_{2}+\lambda \mathrm{x}_{1}$

where $D$ represents the differentiation, and $a$ is the fractional order of the $D$.

$\dot{s}=\mathrm{D}^{\alpha-1} \dot{x}_{2}+\lambda \dot{x}_{1}$

As per Eq. (3),

$0=\mathrm{D}^{\alpha-1} \dot{x}_{2}+\lambda \dot{x}_{1}$

The control force for FOSMC is

$f_{a}=k_{1}\left(y_{1}-y_{2}\right)+c_{1}\left(\dot{y}_{1}-\dot{y}_{2}\right)-D^{\alpha-1} \lambda m_{1}\left(\dot{y}_{1}-\dot{y}_{2}\right)-k \operatorname{sign}(s)$

\section{Results and discussion}

In this section, the simulation results of designed controllers for HDV are studied. The HDV model and the controllers are simulated in the MATLAB/Simulink environment. The parameters used for simulation are chosen by trial and error method. The chosen parameters are $a=0.85, \lambda=0.9$, $K=1$. The performances of the vehicle suspension under step, random and sinusoidal disturbances are investigated with PSS, PID, SMC and FOSMC. The HDV parameters are used for simulation which is listed in Table 1. The HDV runs at a speed of $30 \mathrm{kmph}$. The three different road profiles [11] are shown in Fig. 2. 


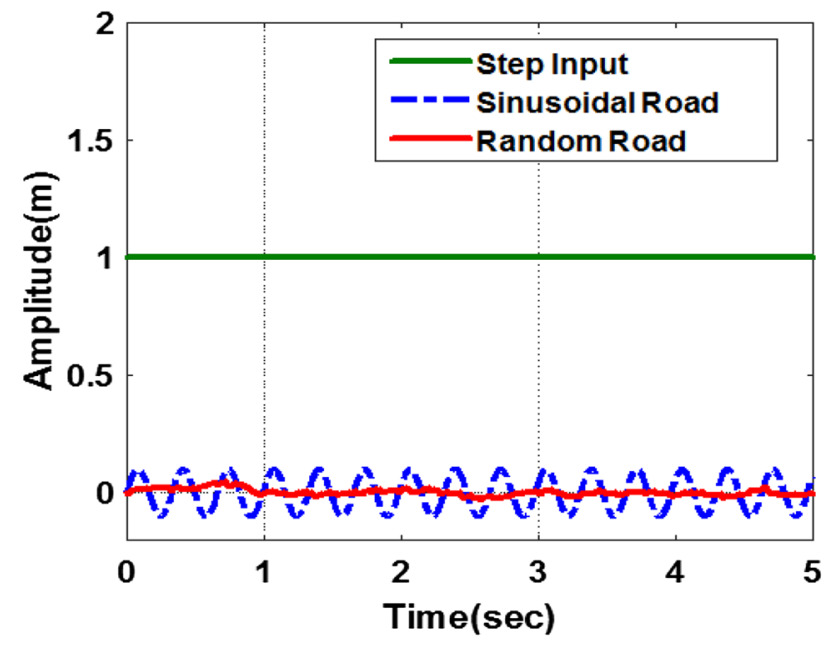

Fig. 2 Road profiles

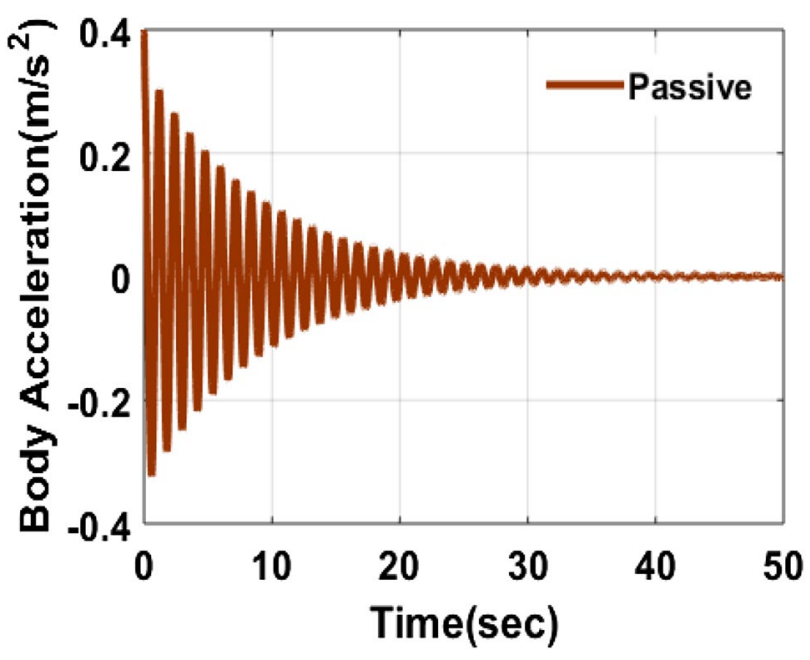

Fig. 3 Passive response of BA-step input

\subsection{Performance analysis of SMCs with step input}

The suppression of BA and SD deteriorate the life span of the ride quality and suspension system of the HDV. For further assessment of potency of the proposed controller with regards to the above mentioned performance, this study is used the Frequency Weighted Root Mean Square (FWRMS) and transient response analysis as performance criteria.

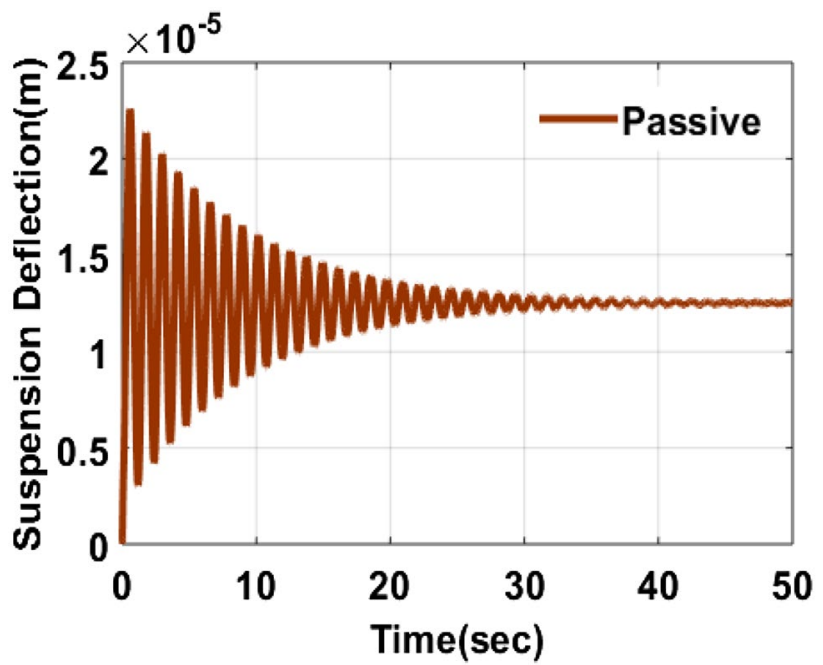

Fig. 4 Passive response of SD-step input

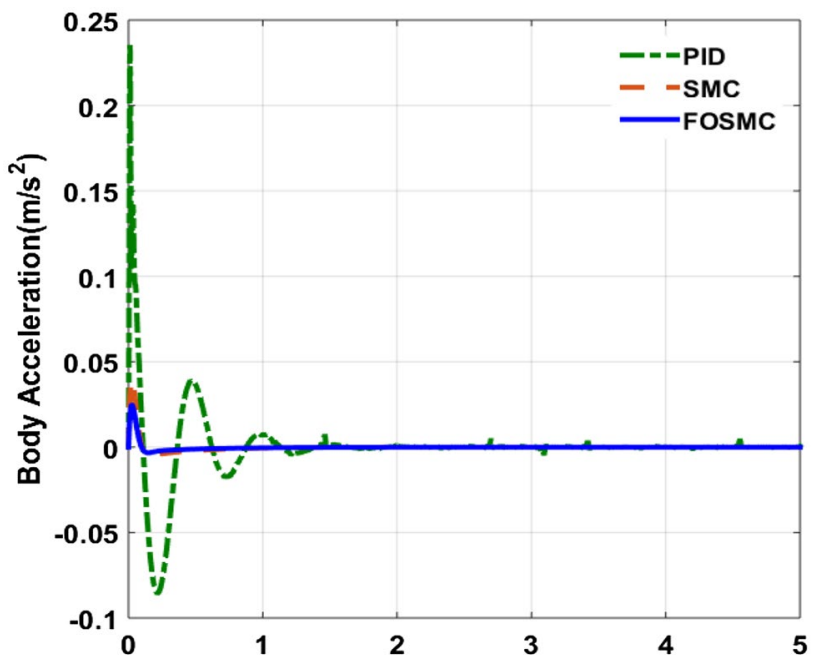

Fig. 5 Active response of BA-step input

The BA and SD of PSS with step input [11] are shown in Figs. 3 and 4 respectively. It is noted from the Fig. 3 , the maximum peak amplitude of $B A$ is $0.4 \mathrm{~m} / \mathrm{s}^{2}$ and settling time is $32 \mathrm{~s}$. Similarly, the maximum peak amplitude and settling time of SD (observed from the Fig. 4) are $2.2 \times 10^{-5} \mathrm{~m}$ and $38 \mathrm{~s}$ respectively. Similarly, the transient responses of ASS with step input are analysed. It can be seen from the Fig. 5, the maximum peak amplitude and 


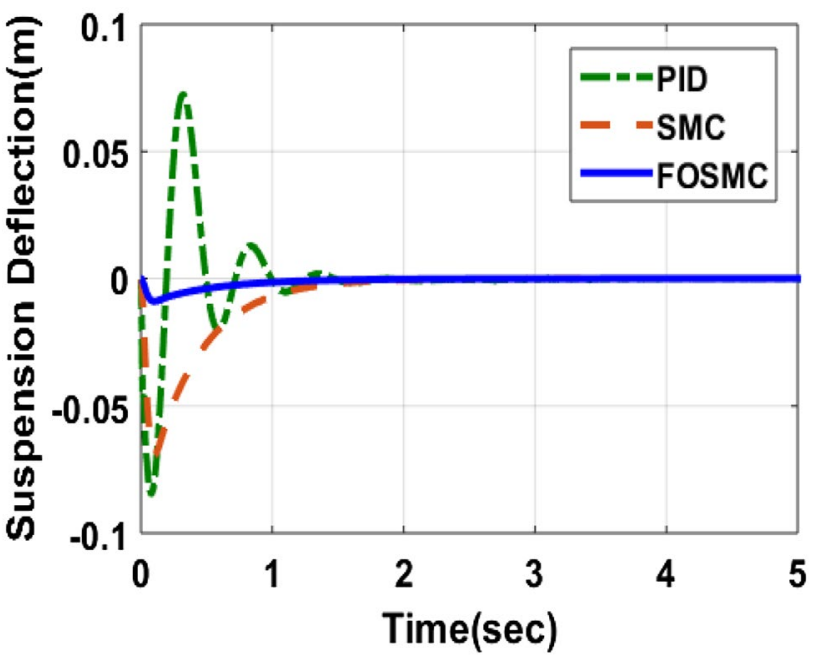

Fig. 6 Active response of SD-step input

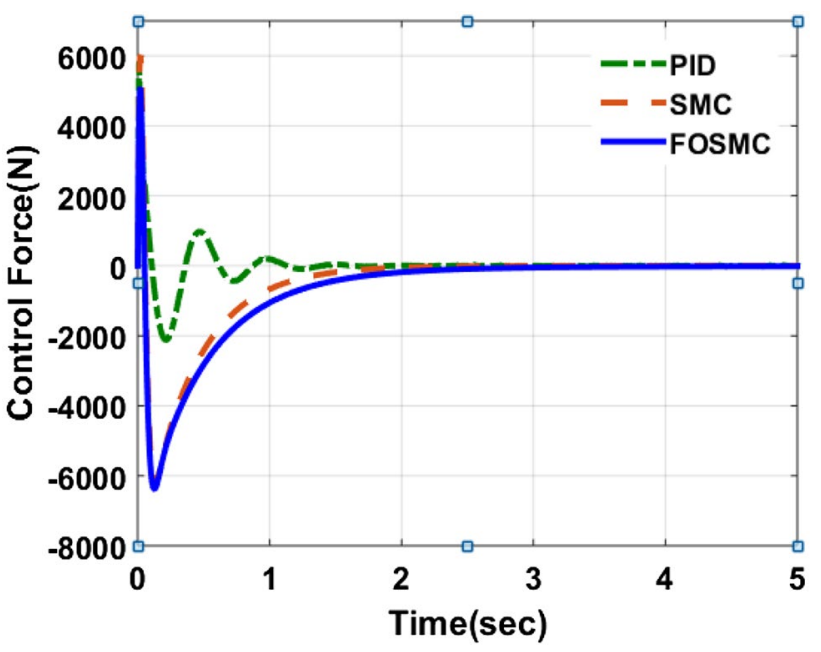

Fig. 7 Control force-step input

settling time of BA with FOSMC are $0.03 \mathrm{~m} / \mathrm{s}^{2}$ and $0.2 \mathrm{~s}$ whereas the BA for SMC are $0.04 \mathrm{~m} / \mathrm{s}^{2}$ and $0.25 \mathrm{~s}$ (Fig. 5).

The settling time of SD (Fig. 6) with FOSMC and SMC are $0.8 \mathrm{~s}$ and $1.2 \mathrm{~s}$. Also the overshoot and settling time of PID are listed in the Table 5. From the abovementioned time domain analysis shows that the maximum peak amplitude and settling time of FOSMC are reduced appreciably than SMC and PID. The control force of the designed controllers is shown in Fig. 7. Form this response; the FOSMC takes more control force to attain desired response.

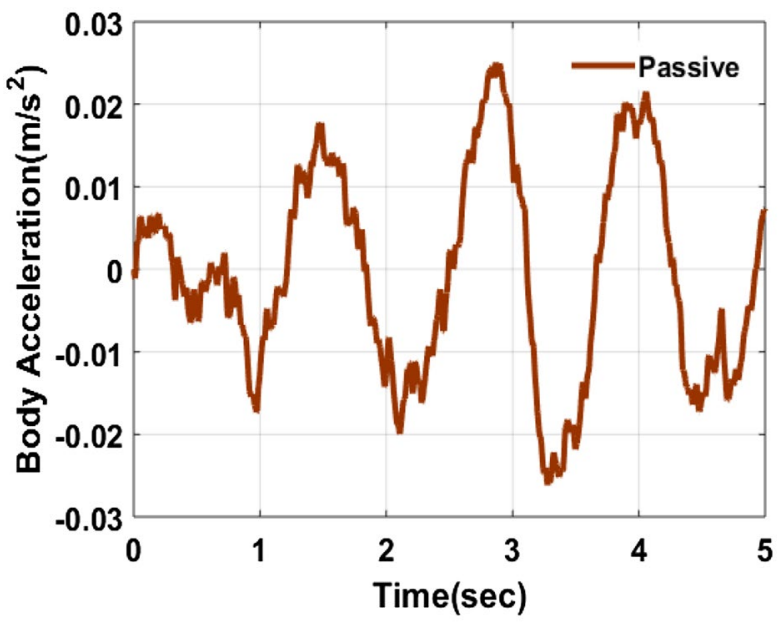

Fig. 8 Passive response of BA-RR input

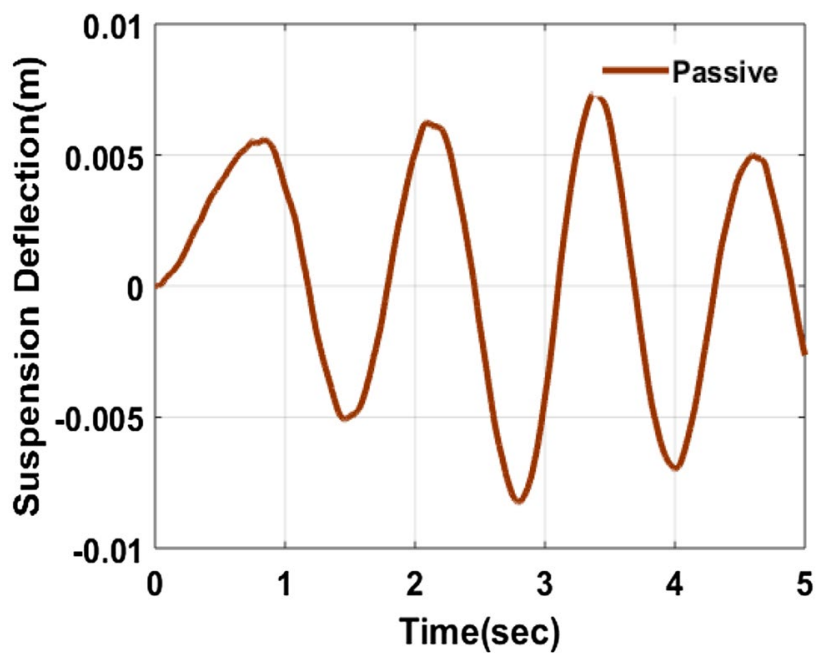

Fig. 9 Passive response of SD-RR input

\subsection{Performance analysis of SMCs with random road}

To evaluate the performances of the SMCs, it is very essential to examine the real road as the disturbance to the HDV model. The real road profile is random road (RR) in nature and it is modelled as per ISO 8608. There are more deformities in the real road and varies continuously with distance. The suspension performances are validated by using RR input.

The SMCs parameters designed for step input is utilised for RR profile. The passive responses of BA and SD are shown in Figs. 8 and 9 respectively [11]. The active responses of $B A$ and SD with RR are shown in Figs. 10 and 11. 


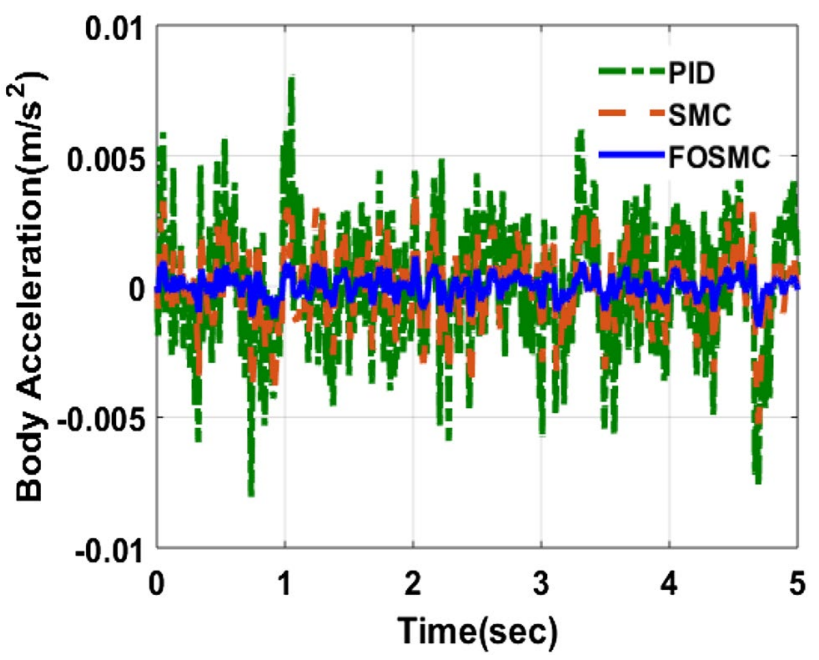

Fig. 10 Active response of BA-RR input

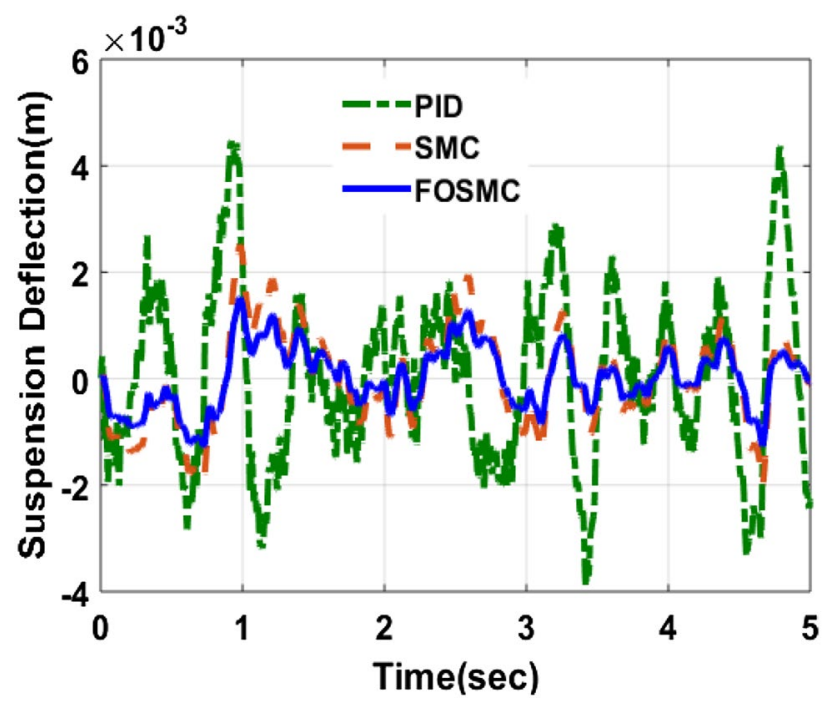

Fig. 11 Active response of SD-RR input

It can be observed from the Figs. 10 and 11, the vibration level of BA and SD with FOSMC is significantly decreased when compared to SMC, PID and PSS. The vibration produced by RR is decreased with FOSMC than PID, SMC and PSS. The corresponding control forces of FOSMC, SMC and PID are shown in Fig. 12.

\subsection{Performance analysis of SMCs with sinusoidal road}

The sinusoidal road (SR) is considered to examine the performances of the designed controllers and it is periodic in nature. Figures 13 and 14 shows the passive response

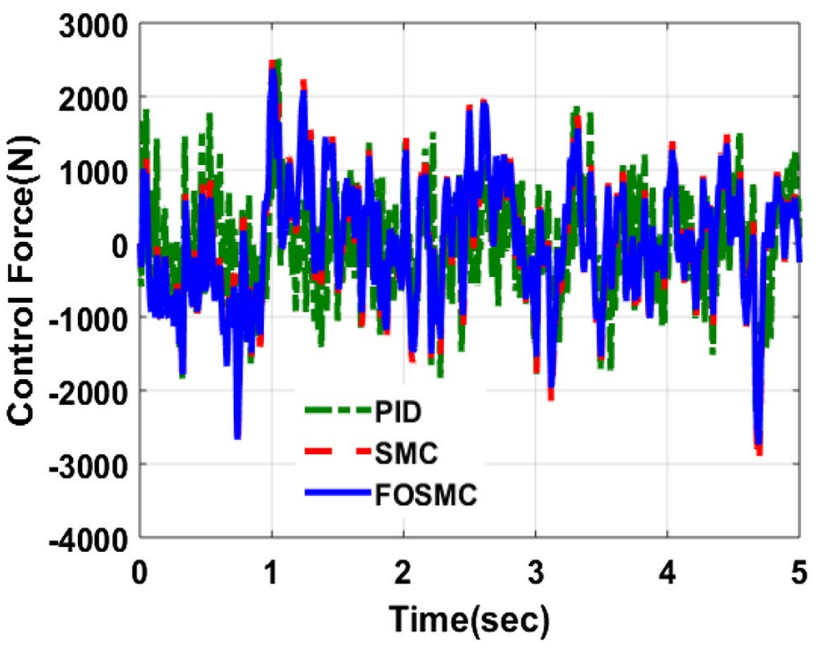

Fig. 12 Control force-RR input

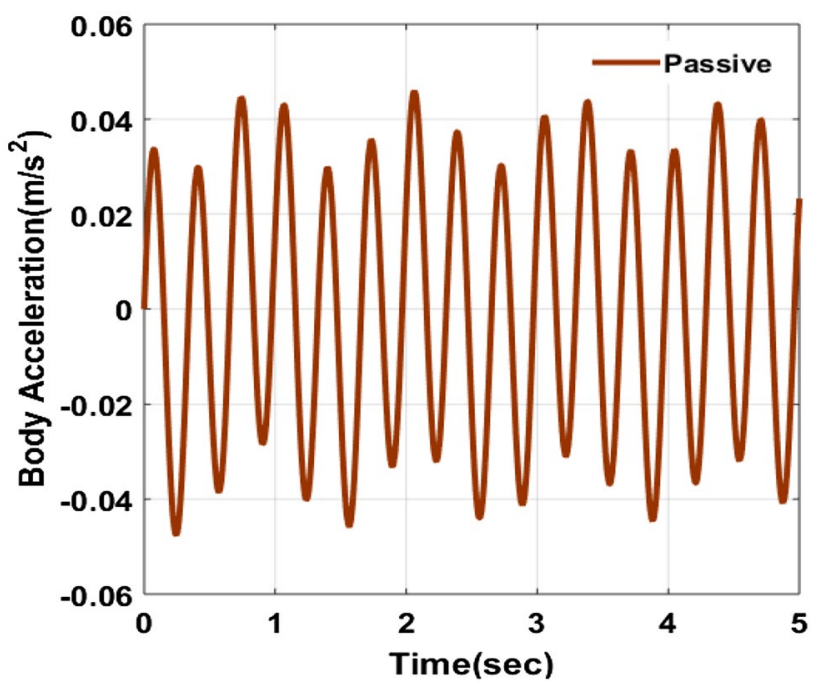

Fig. 13 Passive response of BA-SR input

of HDV model [11]. The active responses of SR input are shown in Figs. 15 and 16.

The corresponding control force is shown in Fig. 17. The magnitude of passive response is increased due to SR. Therefore the more control force is required to reduce the vibration of HDV. The designed controller performances are analysed from the Figs. 15 and 16 and the FOSMC has the effective control over BA and SD. Hence the desirable output can be achieved with FOSMC which enhances the ride comfort by suppressing the vibration due to SR input than SMC, PID and PSS. 


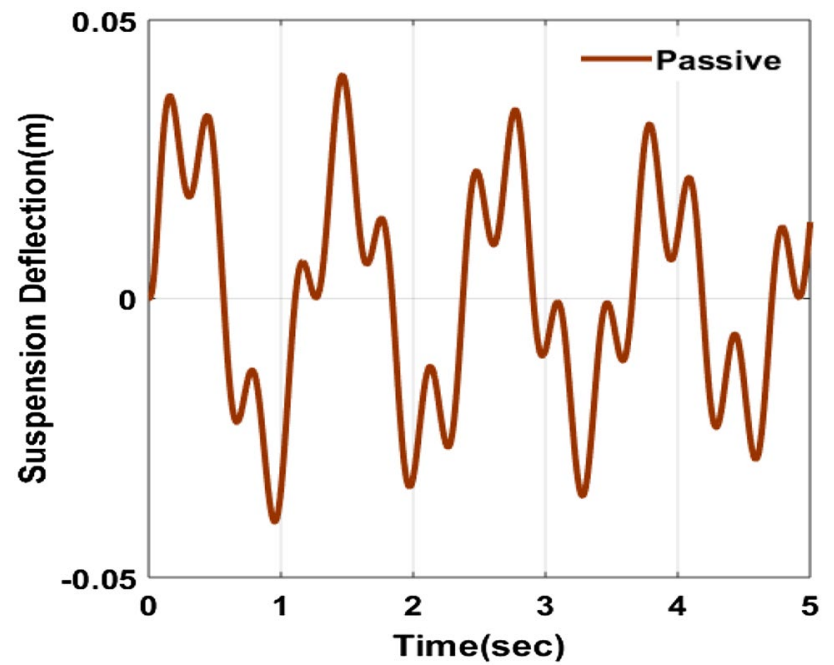

Fig. 14 Passive response of SD-SR input

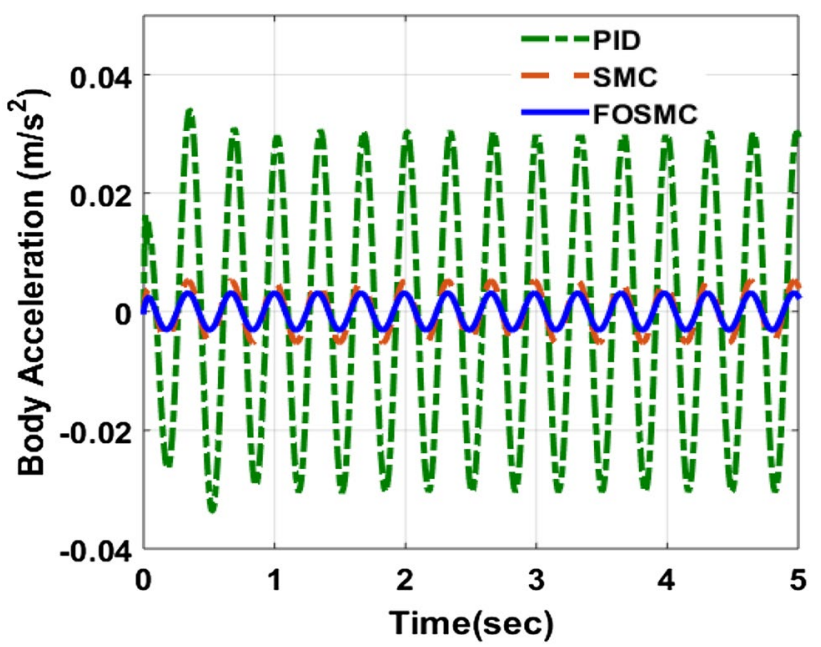

Fig. 15 Active response of BA-RR input

\subsection{Frequency domain and stability analysis}

The linearised model of HDV is analysed in terms of frequency domain using bode plot. The bode plot of BA is plotted for PID and PSS with Step, RR and SR Inputs. It can be observed from the Figs. 18, 19 and 20, the resonant peak of PSS is comparatively higher than PID controller.

The stability of SMC and FOSMC has been proved by Lyapunouv stability criteria for step, RR and SR. The system trajectories are plotted for SMCs with respect to state variables of HDV under step, RR and SR (shown in Figs. 21, 22, $23,24,25$ and 26). The trajectories of SMC and FOSMC are converging to origin for step (Figs. 21, 22) and SR input

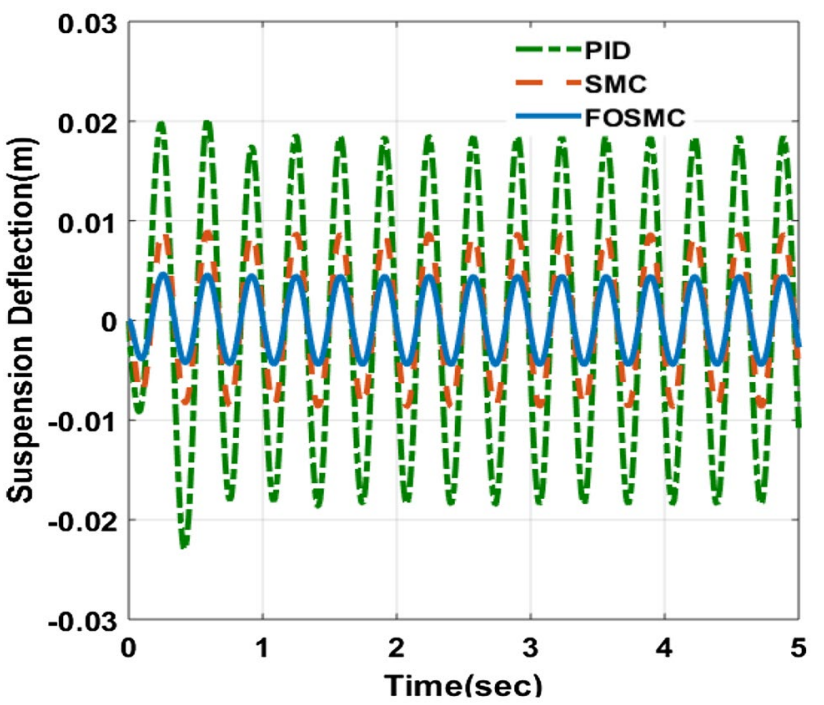

Fig. 16 Active response of SD-SR input

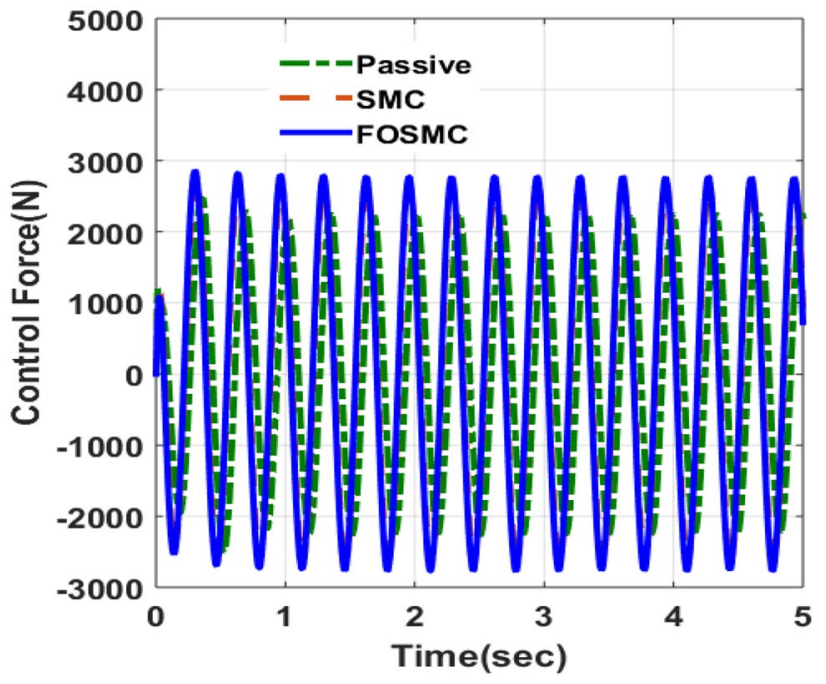

Fig. 17 Control force-SR input

(Figs. 25, 26). Therefore, the FOSMC and SMC for step and SR input are stable as per Lyapunouv stability criteria [26].

Similarly it is tested for RR (Figs. 23, 24) to prove the potential of the designed controllers. The system trajectories neither converge nor diverge to infinity. It indicates the marginal stability of HDV with RR.

\subsection{Ride comfort analysis}

To analyse the ride comfort of the passenger, FWRMS values and vibration dose value (VDV) [27] are computed and it is tabulated in Tables 2, 3 and 4. 


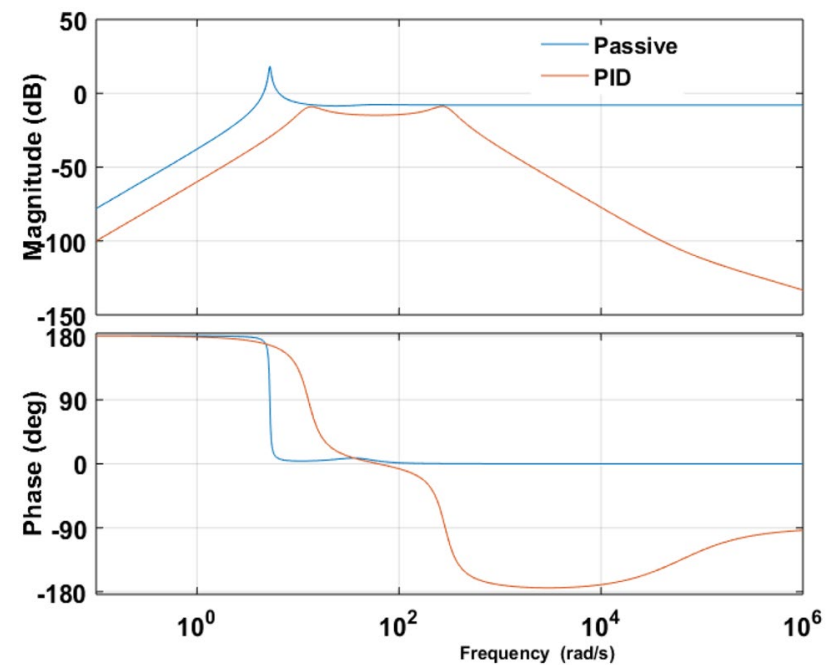

Fig. 18 Frequency response of BA-step input

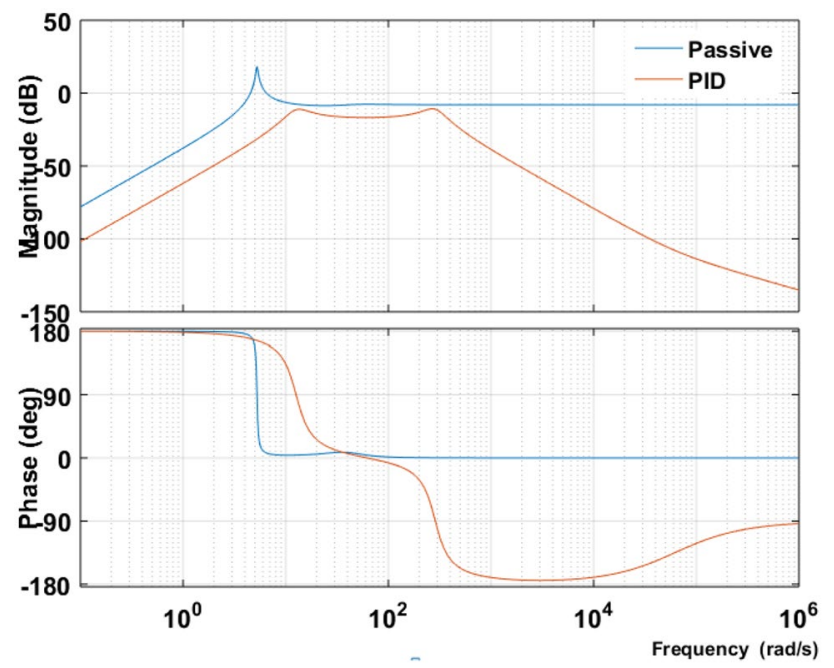

Fig. 19 Frequency response of BA-RR Input

As per ISO-2631 [27], the proposed FOSMC enhances ride comfort to NU level for step input, FU level for RR and LU level for SR input which is noted from the Tables 2, 3 and 4. The FWRMS and vibration dose value (VDV) may cause discomfort for the passengers. Hence the cumulative values of VDV are measured to analyse the intermittent vibration level received over an 8 -h or $16-\mathrm{h}$ period. The unit

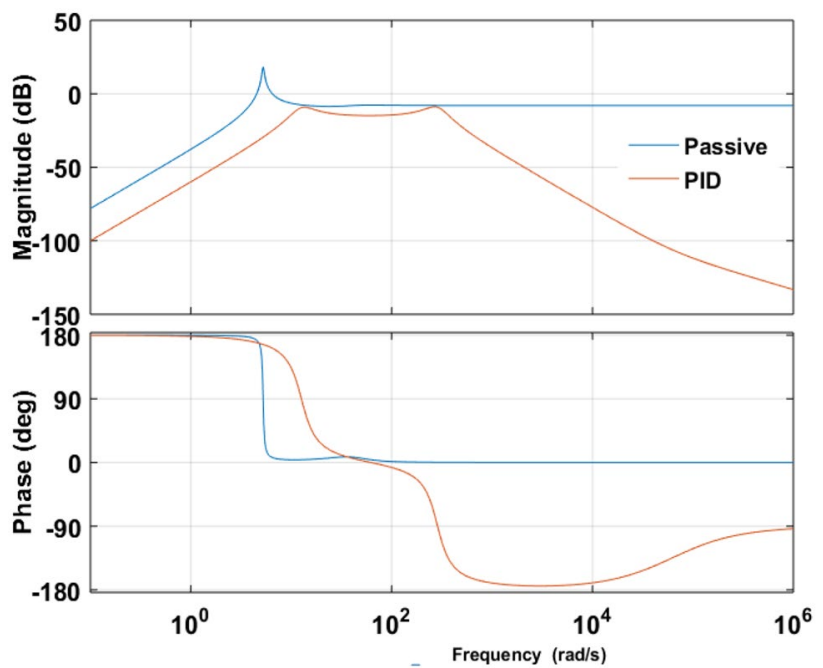

Fig. 20 Frequency response of BA-SR input

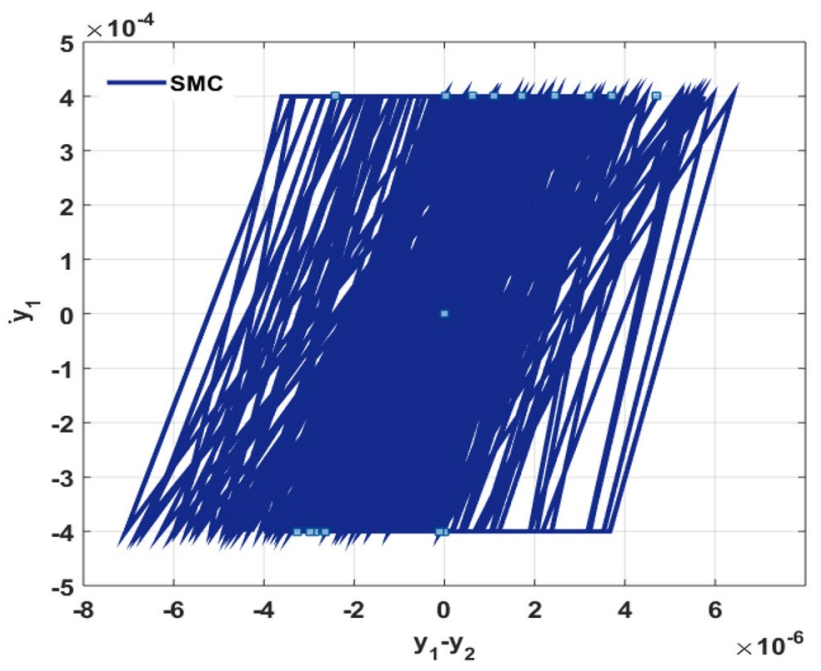

Fig. 21 Phase plane of SMC-step input

of VDV is taken from safety sciences. The ISO2631-1 Standard uses the exposure duration and Vibration Dose Values (VDV) to calculate the equivalence, being those terms a power of 2 or 4 , respectively, in the equivalence formula [27]. It is evaluated for step, RR and SR input. Hence the numerical values of FWRMS and VDV are decreased for 


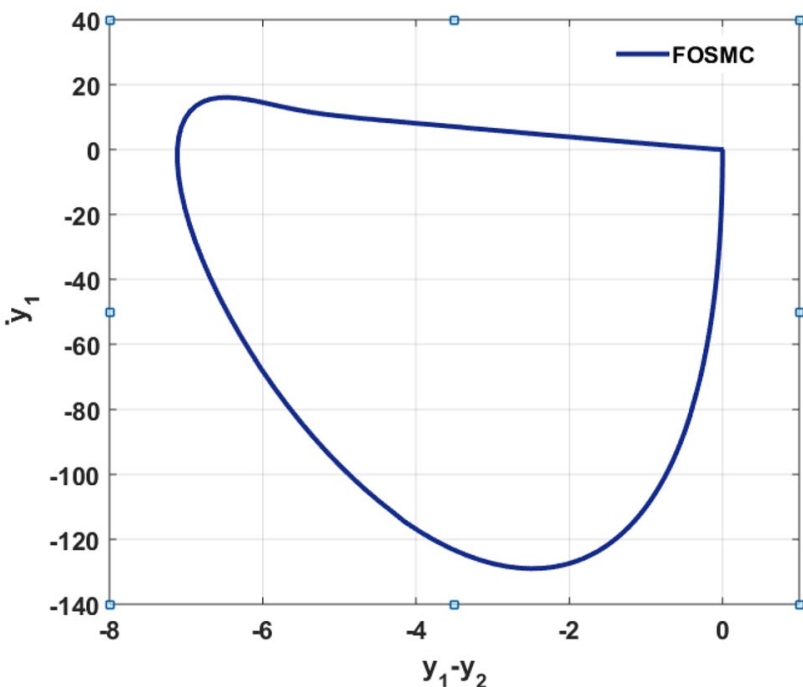

Fig. 22 Phase plane of FOSMC-step input

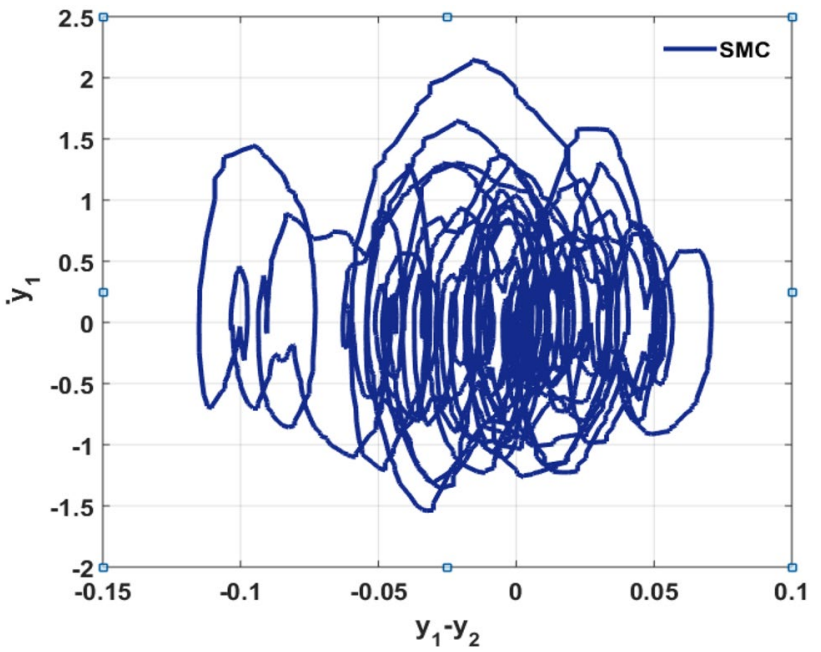

Fig. 23 Phase plane of SMC-RR input

FOSMC when compared to SMC and PID and it improves the quality of ride comfort.

\subsection{Power spectrum density analysis}

The human sensitivity range lies in the range of $4-8 \mathrm{~Hz}$. It is observed from the Figs. 27, 28 and 29, the designed FOSMC lies in the human sensitivity range than PSS. Hence, the frequency range of PSD of BA with FOSMC improves the ride comfort of the passenger for three different road profiles.

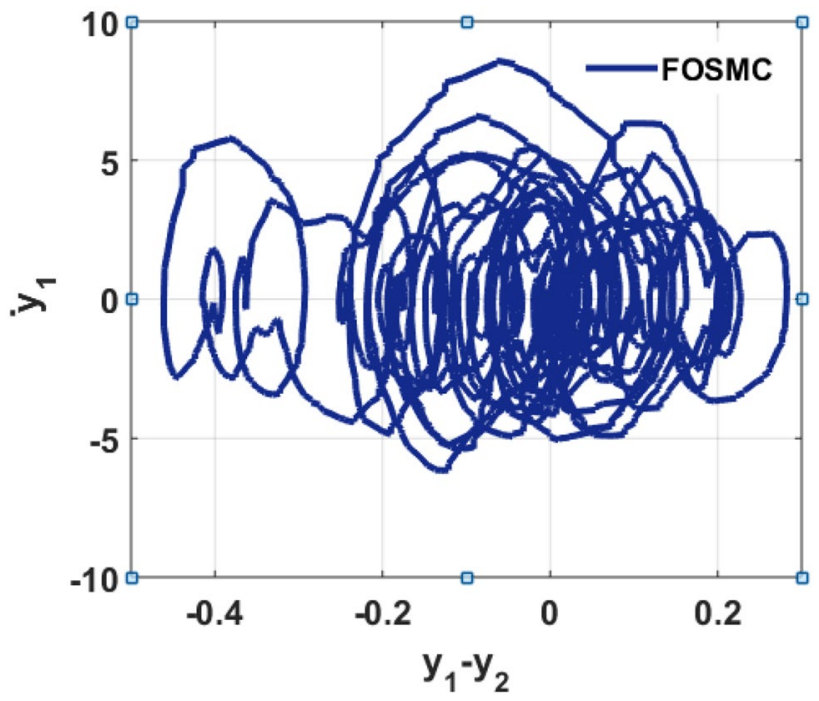

Fig. 24 Phase plane of FOSMC-RR

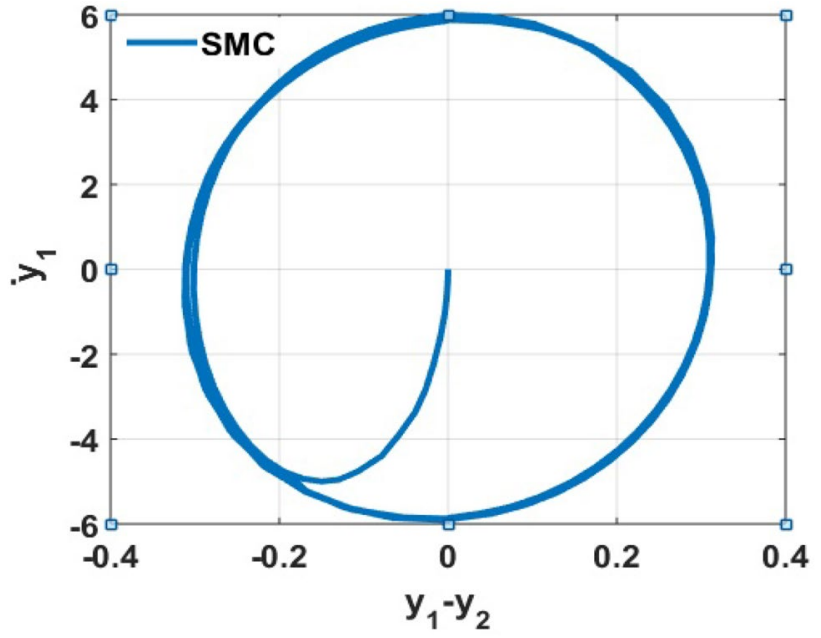

Fig. 25 Phase plane of SMC-SR input

\subsection{Comparative analysis with existing results}

The comparative analysis of HDV is made with existing PID [22] controller. The time responses of designed FOSMC and SMC are compared with existing PID controller with step input and the time domain specifications are listed in Table 5.

It can be observed from the Table 5, the proposed FOSMC settles quickly within $1 \mathrm{~s}$ and there is no overshoot compared to existing PID controller. The numerical and simulation results prove that the efficacy of the proposed FOSMC than existing PID controller. 


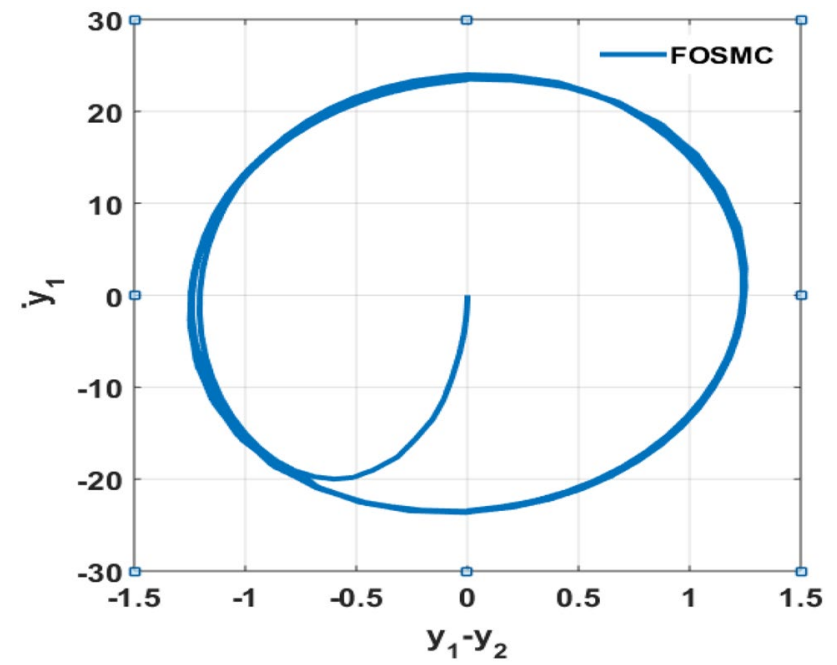

Fig. 26 Phase plane of FOSMC-SR input

Table 2 Ride comfort analysis with step input

\begin{tabular}{llll}
\hline Controllers & \multicolumn{3}{l}{ Step input } \\
\cline { 2 - 4 } & BA $\left(\mathrm{rad} / \mathrm{s}^{2}\right)$ & UL & VDV $\left(\mathrm{m} / \mathrm{s}^{1.75}\right)$ \\
\hline PSS & 1.82 & VU & 2.13 \\
PID & 1.423 & U & 1.75 \\
SMC & 0.745 & FU & 1.1 \\
FOSMC & 0.296 & NU & 0.636 \\
\hline
\end{tabular}

Extremely uncomfortable (EU), very uncomfortable (VU), uncomfortable (U), not uncomfortable (NU), not uncomfortable (NU), little uncomfortable (LU), fairly uncomfortable (FU)

Table 3 Ride comfort analysis with RR input

\begin{tabular}{llll}
\hline Controllers & \multicolumn{2}{l}{ RR input } & \\
\cline { 2 - 4 } & BA $\left(\mathrm{rad} / \mathrm{s}^{2}\right)$ & UL & VDV $\left(\mathrm{m} / \mathrm{s}^{1.75}\right)$ \\
\hline PSS & 2.52 & EU & 2.82 \\
PID & 1.81 & VU & 2.11 \\
SMC & 1.2 & U & 1.53 \\
FOSMC & 0.95 & FU & 1.31 \\
\hline
\end{tabular}

Table 4 Ride comfort analysis with SR input

\begin{tabular}{llll}
\hline Controllers & \multicolumn{3}{l}{ SR input } \\
\cline { 2 - 4 } & BA $\left(\mathrm{rad} / \mathrm{s}^{2}\right)$ & UL & VDV $\left(\mathrm{m} / \mathrm{s}^{1.75}\right)$ \\
\hline PSS & 1.98 & VU & 2.61 \\
PID & 1.31 & U & 1.73 \\
SMC & 0.75 & FU & 1.12 \\
FOSMC & 0.33 & LU & 0.632 \\
\hline
\end{tabular}

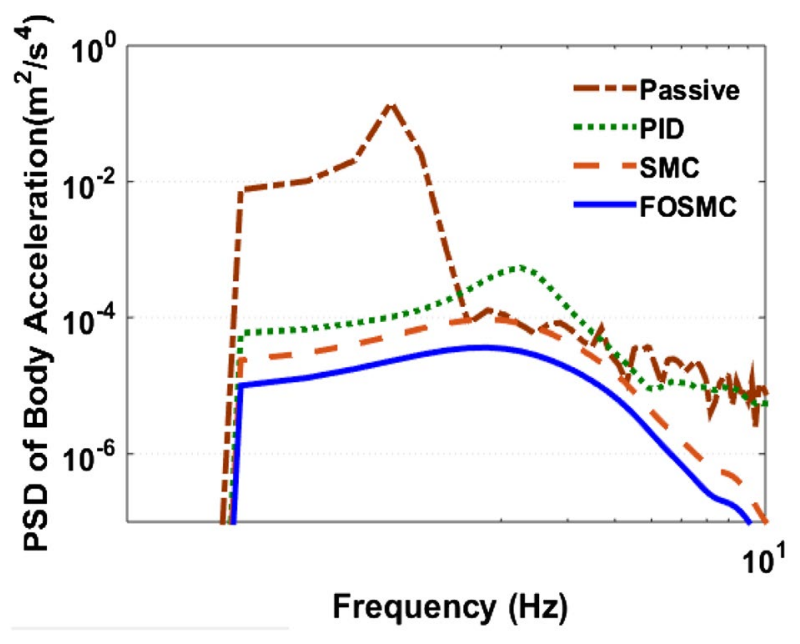

Fig. 27 PSD of BA-step input

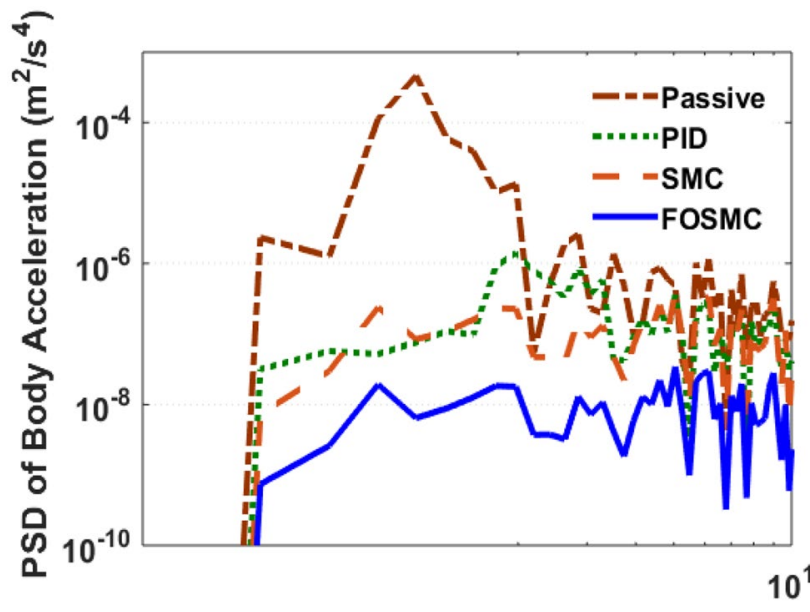

Frequency $(\mathrm{Hz})$

Fig. 28 PSD of BA-RR input

\section{Conclusion}

In this paper, the FOSMC is proposed for HDV with ASS. The 2 DOF HDV linear model is considered for controller design. The Proposed FOSMC can suppress the BA and SD while travelling over the step, RR and SR. The FWRMS and VDV are computed based on ISO-2631 to confirm the effectiveness of the proposed FOSMC. The time domain analysis of HDV with PSS, PID, SMC and FOSMC is validated using MATLAB/Simulink software. The step, RR and $S R$ inputs are taken for the investigation of the FOSMC. The simulation results with step input is compared to existing PID controller which proves the effectiveness of the FOSMC than PID, SMC and PSS. Further discussion 


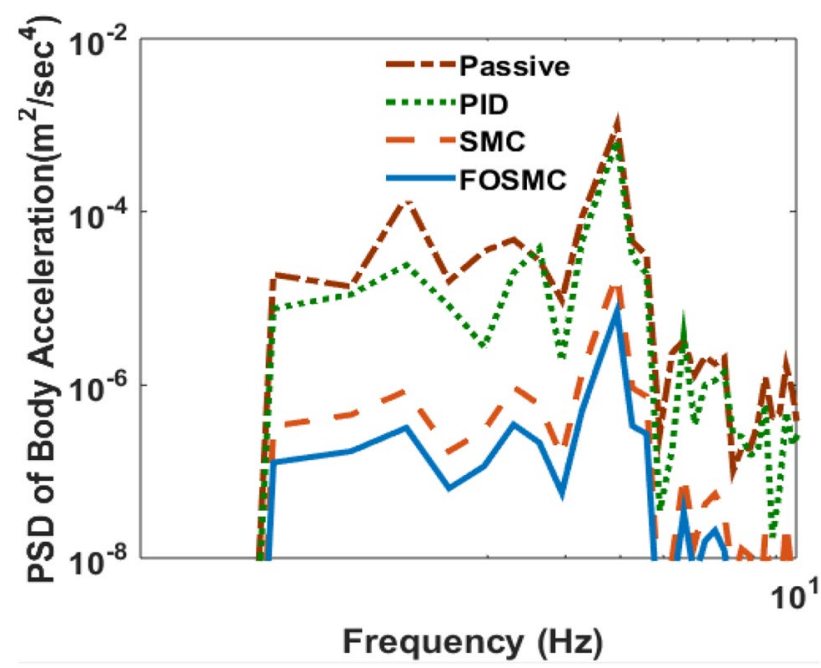

Fig. 29 PSD of BA-SR input

Table 5 Time domain analysis

\begin{tabular}{lll}
\hline Controllers & \multicolumn{2}{l}{ Suspension deflection $(\mathrm{m})$} \\
\cline { 2 - 3 } & Overshoot $(\mathrm{m})$ & $\begin{array}{l}\text { Settling } \\
\text { time }(\mathrm{s})\end{array}$ \\
\hline PSS & $2.2 \times 10^{-5}$ & 38 \\
PID & 0.81 & 2 \\
SMC & - & 1.2 \\
FOSMC & - & 0.9 \\
\hline
\end{tabular}

confirms that the stability of the vehicle is guaranteed by FOSMC and SMC.

\section{Compliance with ethical standards}

Conflict of interest On behalf of all authors, the corresponding author states that there is no conflict of interest.

\section{References}

1. Sert E, Boyraz P (2017) Optimization of suspension system and sensitivity analysis for improvement of stability in a midsize heavy vehicle. Eng Sci Technol Int J 20(3):997-1012

2. Zhang J, Deng Y, Zhang N, Zhang B, Qi H, Zheng M (2019) Vibration performance analysis of a mining vehicle with bounce and pitch tuned hydraulically interconnected suspension. Chin J Mech Eng 32(1):5

3. Ieluzzi M, Turco P, Montiglio M (2006) Development of a heavy truck semi-active suspension control. Control Eng Pract 14(3):305-312

4. Pazooki A, Rakheja S, Cao D (2012) Modeling and validation of off-road vehicle ride dynamics. Mech Syst Signal Process 28:679-695
5. Mazurek B, Mamala J, Miozga R (2018) Mechatronic damping ratio control system of the progressive shock absorber in offroad vehicle. In: AIP conference proceedings, vol 2029, no 1. AIP Publishing LLC, p 020040

6. Ning D, Sun S, Zhang J, Du H, Li W, Wang X (2016) An active seat suspension design for vibration control of heavy-duty vehicles. J Low Freq Noise Vib Active Control 35(4):264-278

7. EIMadany MM, Abduljabbar Z (1990) Design evaluation of advanced suspension systems for truck ride comfort. Comput Struct 36(2):321-331

8. Imine H, Fridman LM, Madani T (2012) Steering control for rollover avoidance of heavy vehicles. IEEE Trans Veh Technol 61(8):3499-3509

9. Ryu YI, Kang DO, Heo SJ, In JH (2010) Rollover mitigation for a heavy commercial vehicle. Int J Automot Technol 11(2):283-287

10. Vaughan J, Singhose W, Sadegh N (2003) Use of active suspension control to counter the effects of vehicle payloads. In: Proceedings of 2003 IEEE conference on control applications, 2003. CCA 2003, vol 1. IEEE, pp 285-289

11. Yuvapriya T, Lakshmi P (2020) Numerical analysis and performance enhancement of active suspension system using bat optimization. Int J Dyn Control. https://doi.org/10.1007/s4043 5-020-00664-5

12. He F, Zhao J (2011) Simulation study on ride comfort and roadfriendliness of heavy vehicles based on multi-objective optimization. In: Proceedings 2011 international conference on transportation, mechanical, and electrical engineering (TMEE). IEEE, pp 1293-1296

13. Zhao Q, Yang Y (2008) Improved single neuron PID control for heavy-duty vehicle magnetorheological seat suspension. In: 2008 IEEE vehicle power and propulsion conference. IEEE, pp $1-3$

14. Ansari FA, Taparia RS (2013) Modeling, analysis and control of active suspension system using sliding mode control and disturbance observer. Int J Sci Res Publ 3(1):1-6

15. Yuvapriya T, Lakshmi $P$ (2019) Design of fractional order sliding mode controller for semi active suspension system. In: 2019 IEEE international systems conference (SysCon). IEEE, pp 1-5

16. Rajendiran S, Lakshmi P, Rajkumar B (2018) Enhancing the travel comfort of quarter-car model using fractional order terminal sliding mode controller. J Adv Veh Eng 4(4):141-149

17. Yuvapriya T, Lakshmi P, Rajendiran S (2020) Vibration control and performance analysis of full car active suspension system using fractional order terminal sliding mode controller. Arch Control Sci 30:295-324. https://doi.org/10.24425/acs.2020.133501

18. Yadav AK, Gaur P (2017) Speed control of an uncertain heavyduty vehicle using improved IMC technique. Arab J Sci Eng 42(7):2981-2991

19. Eski İ, Yıldırım Ş (2017) Neural network-based fuzzy inference system for speed control of heavy duty vehicles with electronic throttle control system. Neural Comput Appl 28(1):907-916

20. Shin CW, Kim H, Kim MK, Lim W, Cha SW (2014) Development of an evaluation method for quantitative driveability in heavy-duty vehicles. J Mech Sci Technol 28(5):1615-1621

21. Tahir A, Yasin J, Daneshtalab M, Plosila J (2014) Active suspension system for heavy vehicles. In: 2014 IEEE international symposium on fundamentals of electrical engineering (ISFEE), pp $1-5$

22. Gaur S, Jain $S$ (2013) Vibration control of bus suspension system using PI and PID controller. Int J Adv Eng Sci 3(3):94-99

23. Coughanowr DR, Koppel LB (1965) Process systems analysis and control, vol 2. McGraw-Hill, New York

24. Yuvapriya T, Lakshmi P, Rajendiran S (2018) Vibration suppression in full car active suspension system using fractional order sliding mode controller. J Braz Soc Mech Sci Eng 40(4):217 
25. Yuvapriya T, Lakshmi P, Rajendiran S (2020) Design, analysis and experimental validation of a fractional order terminal sliding mode controller on active suspension system. IETE J Res. https ://doi.org/10.1080/03772063.2020.1756472

26. Slotine JJE, Li W (1991) Applied nonlinear control, no. 1, vol 199. Prentice Hall, Englewood Cliffs

27. Duarte MLM, de Araújo PA, Horta FC, Del Vecchio S, de Carvalho LAP (2018) Correlation between weighted acceleration, vibration dose value and exposure time on whole body vibration comfort levels evaluation. Saf Sci 103:218-224

Publisher's Note Springer Nature remains neutral with regard to jurisdictional claims in published maps and institutional affiliations. 